\title{
A criterion for $p$-henselianity in characteristic $p$
}

\author{
Zoé Chatzidakis*and Milan Perera
}

\begin{abstract}
Let $p$ be a prime. In this paper we give a proof of the following result: A valued field $(K, v)$ of characteristic $p>0$ is $p$-henselian if and only if every element of strictly positive valuation if of the form $x^{p}-x$ for some $x \in K$.
\end{abstract}

\section{Preliminaries}

Throughout this paper, all fields have characteristic $p>0$. First we recall some definitions and notations. Let $\mathcal{O}_{v}:=\{x \in K \mid v(x) \geq 0\}$ be the valuation ring associated with $v$. It is a local ring, and $\mathcal{M}_{v}:=\{x \in K \mid$ $v(x)>0\}$ is its maximal ideal. Let $\bar{K}_{v}:=\mathcal{O}_{v} / \mathcal{M}_{v}=\left\{\bar{a}=a+\mathcal{M}_{v} \mid a \in \mathcal{O}_{v}\right\}$ be the residue field (or $\bar{K}$ when there is no danger of confusion). We let $K(p)$ denote the compositum of all finite Galois extensions of $K$ of degree a power of $p$.

A valued field $(K, v)$ is $p$-henselian if $v$ extends uniquely to $K(p)$. Equivalently (see [1], Thm 4.3.2), if it satisfies a restricted version of Hensel's lemma (which we call $p$-Hensel lemma) : $K$ is $p$-henselian if and only if every polynomial $P \in \mathcal{O}_{v}[X]$ which splits in $K(p)$ and with residual image in $\bar{K}_{v}[X]$ having a simple root $\alpha$ in $\bar{K}_{v}$, has a root $a$ in $\mathcal{O}_{v}$ with $\bar{a}=\alpha$. Furthermore, another result (see [1], Thm 4.2.2) shows that $(K, v)$ is $p$-henselian if and only if $v$ extends uniquely to every Galois extension of degree $p$.

The aim of this note is to give a complete proof of the following result:

Theorem. Let $(K, v)$ be a valued field. $(K, v)$ is $p$-henselian if and only if $\mathcal{M}_{v} \subseteq\left\{x^{p}-x \mid x \in K\right\}$.

\footnotetext{
*Partially supported by ANR-13-BS01-0006
} 
This result was announced in [3], Proposition 1.4, however the proof was not complete. The notion of $p$-henselianity is important in the study of fields with definable valuations, and in particular it is important to show that the property of $p$-henselianity is an elementary property of valued fields.

The proof we give is elementary, and uses extensively pseudo-convergent sequences and their properties. Recall that a sequence $\left\{a_{\rho}\right\}_{\rho<\kappa} \in K^{\kappa}$ indexed by an ordinal $\kappa$ is said to be pseudo-convergent if for all $\alpha<\beta<\gamma<\kappa$ :

$$
v\left(a_{\beta}-a_{\alpha}\right)<v\left(a_{\gamma}-a_{\beta}\right) .
$$

A pseudo-convergent sequence $\left\{a_{\rho}\right\}_{\rho<\kappa}$ is called algebraic if there is a polynomial $P$ in $K[X]$ such that $v\left(P\left(a_{\alpha}\right)\right)<v\left(P\left(a_{\beta}\right)\right)$ ultimately for all $\alpha<\beta$, i.e:

$$
\exists \lambda<\kappa \forall \alpha, \beta<\kappa \quad(\lambda<\alpha<\beta) \Rightarrow v\left(P\left(a_{\alpha}\right)\right)<v\left(P\left(a_{\beta}\right)\right) .
$$

Otherwise, it is called transcendental.

We assume familiarity with the properties of pseudo-convergent sequences, see [2] for more details, and in particular Theorem 3, Lemmas 4 and 8.

\section{Proof of the theorem}

First, we prove a lemma in order to restrict our study to immediate extensions:

Observation. Let $(K, v)$ be a valued field and $(L, w)$ be a Galois extension of degree a prime $\ell$. Then, if $(L, w) /(K, v)$ is residual or ramified, $w$ is the unique extension of $v$ to $L$.

Proof. The fundamental equality of valuation theory (see [1], Thm 3.3.3) tells us that if $L$ is a Galois extension of $K$, then

$$
[L: K]=e(L / K) f(L / K) g d
$$

where $e(L / K)$ is the ramification index, $f(L / K)$ the residue index, $g$ the number of extensions of $v$ to $L$ and $d$, the defect, is a power of $p$.

Thus, as $\ell$ is a prime, if $e(L / K) f(L / K)>1$, then necessarily $g=d=1$, and in particular, $v$ has a unique extension to $L$. 
Now, let us prove the result announced in the preliminaries:

Theorem. Let $\left(K, \mathcal{O}_{v}\right)$ be a valued field of characteristic $p$. Then, $\left(K, \mathcal{O}_{v}\right)$ is $p$-henselian if and only if $\mathcal{M}_{v} \subseteq K^{(p)}:=\left\{x^{p}-x \mid x \in K\right\}$.

Proof. The forward direction is an immediate application of the $p$-Hensel Lemma.

Conversely, assume that $\mathcal{M}_{v} \subseteq K^{(p)}:=\left\{x^{p}-x \mid x \in K\right\}$. Every Galois extension of $K$ of degree $p$ is an Artin-Schreier extension, i.e is generated over $K$ by a root $a$ of a polynomial $X^{p}-X-b=0$, with $b \in K \backslash K^{(p)}$. The previous observation gives us the result when $K(a) / K$ is not immediate. Let $L$ be an immediate Galois extension of degree $p$ and $\tilde{v}$ an extension of $v$ to $L$ (hence with the same value group $\Gamma$ and residue field $\bar{L}=\bar{K}$ as $K$ ). We can write $L=K(a)$ where $a^{p}-a=b \in K \backslash K^{(p)}$.

Step 1: (Claim) The set $C=\left\{v\left(x^{p}-x-b\right) \mid x \in K\right\}=v\left(K^{(p)}-b\right)$ is contained in $\Gamma_{<0}$ and has no last element.

First observe that $C \subseteq \Gamma_{\leq 0}$ : if $v\left(c^{p}-c-b\right)>0$, then the equation $X^{p}-X+\left(c^{p}-c-b\right)$ has a root in $K$, so that $(a-c) \in K$ : contradiction. Let $\gamma \in \Gamma, d \in K$ such that $v\left(d^{p}-d-b\right)=\gamma$. As $L / K$ is immediate there is $c \in K$ such that $\tilde{v}(a-(d+c))>\tilde{v}(a-d)$. If $\tilde{v}(a-d)=0$ then $\tilde{v}(a-(d+c))>0$ and $\left((d+c)^{p}-(d+c)-b\right)=(d+c-a)^{p}-(d+c-a)$ in $\mathcal{M}_{v}$, which as above give a contradiction. Hence $\tilde{v}(a-d)<0$, and from $d^{p}-d-b=(d-a)^{p}-(d-a)$, we deduce that $\gamma=p \tilde{v}(a-d)<0$, and $v\left((d+c)^{p}-(d+c)-b\right)=p(\tilde{v}(a-(d+c)))>\gamma$. This shows the claim.

Step 2: We extract a strictly well-ordered increasing and cofinal sequence from $C$. If we write $P(X):=X^{p}-X-b$, we get a sequence $\left\{a_{\rho}\right\}_{\rho<\kappa}$ in $K$ such that the sequence $\left\{v\left(P\left(a_{\rho}\right)\right)\right\}_{\rho<\kappa}$ is stricly increasing and cofinal in $C$. Thus, the sequence $\left\{P\left(a_{\rho}\right)\right\}_{\rho<\kappa}$ is pseudo-convergent (with 0 one of its limits). As $v\left(P\left(a_{\alpha}\right)\right)<0$, we have $v\left(a_{\beta}-a_{\alpha}\right)=\frac{1}{p} v\left(P\left(a_{\alpha}\right)\right)=\gamma_{\alpha}$ for $\alpha<\beta<\kappa$. Thus, the sequence $\left\{a_{\rho}\right\}_{\rho<\kappa}$ is also pseudo-convergent. Furthermore, $\left\{a_{\rho}\right\}_{\rho<\kappa}$ has no limit in $K$ : if $l \in K$ is a limit of $\left\{a_{\rho}\right\}_{\rho<\kappa}$ then $P(l)$ is a limit of $\left\{P\left(a_{\rho}\right)\right\}_{\rho<\kappa}$. As $\left\{v\left(P\left(a_{\rho}\right)\right)\right\}_{\rho<\kappa}$ is cofinal in $C, v(P(l))$ would be a maximal element of $C$ : contradiction.

Step 3: (Claim) Let $P_{0}(X) \in K[X]$, and assume that $v\left(P_{0}\left(a_{\alpha}\right)\right)$ is strictly increasing ultimately. Then $\operatorname{deg}\left(P_{0}(X)\right) \geq p$.

We take such a $P_{0}$ of minimal degree, assume this degree is $n<p$, and will 
derive a contradiction. One consequence of Lemma 8 in [2] is that:

$$
v\left(P_{0}\left(a_{\rho}\right)\right)=\delta^{\prime}+\gamma_{\rho} \text { ultimately for } \rho<\kappa
$$

where $\delta^{\prime}$ is the ultimate valuation of $P_{0}^{\prime}\left(a_{\rho}\right)$ and $\gamma_{\rho}$ is the valuation of $\left(a_{\sigma}-a_{\rho}\right)$ for $\rho<\sigma<\kappa$ (which does not depend on $\sigma$ as $\left\{a_{\rho}\right\}_{\rho<\kappa}$ is pseudo-convergent). We write $P(X)=\sum_{i=0}^{m} h_{i}(X) P_{0}(X)^{i}$ with $\operatorname{deg}\left(h_{i}\right)<n, \forall i \in\{1, \ldots, m\}$. Then, $\left\{h_{i}\left(a_{\rho}\right)\right\}_{\rho<\kappa}$ is ultimately of constant valuation, and we let $\lambda_{i}$ be this valuation. As $\left\{a_{\rho}\right\}_{\rho<\kappa}$ has no limit in $K$, it is easy to see that $n>1$, so that $m<p$. By Lemma 4 in [2], there is an integer $i_{0} \in\{1, \ldots, m\}$ such that we have ultimately:

$$
\forall i \neq i_{0} \quad\left(\lambda_{i}+i \delta^{\prime}\right)+i \gamma_{\rho}>\left(\lambda_{i_{0}}+i_{0} \delta^{\prime}\right)+i_{0} \gamma_{\rho}
$$

Then, ultimately:

$$
p \gamma_{\rho}=v\left(P\left(a_{\rho}\right)\right)=v\left(\sum_{i=0}^{m} h_{i}\left(a_{\rho}\right) P_{0}\left(a_{\rho}\right)^{i}\right)=\lambda_{i_{0}}+i_{0}\left(\delta^{\prime}+\gamma_{\rho}\right) .
$$

Thus, we have ultimately $\left(p-i_{0}\right) \gamma_{\rho}=\lambda_{i_{0}}+i_{0} \delta^{\prime}$. As $p>m \geq i_{0}$, the left hand side of the equation increases strictly monotonically with $\rho$. But the right hand side is constant: it has no dependence in $\rho$ ! We have a contradiction, thus $n=p$.

Step 4: Clearly, $\left\{a_{\rho}\right\}_{\rho<\kappa}$ is of algebraic type. By Theorem 3 in [2], if $a_{\infty}$ is a root of $P$, we get an immediate extension $\left(L^{\prime}, v^{\prime}\right)=\left(K\left(a_{\infty}\right), v^{\prime}\right)$. Let $a_{\infty}=a$, we have $\left(K(a), v^{\prime}\right)$ isomorphic to $(K(a), \tilde{v})$. Thus:

$$
\forall Q \in K_{p}[X] \quad \tilde{v}(Q(a))=v^{\prime}(Q(a))=v\left(Q\left(a_{\rho}\right)\right) \text { ultimately }
$$

This shows the uniqueness of $\tilde{v}$ and concludes the proof of the theorem.

\section{References}

[1] A.J. Engler, A. Prestel, Valued fields. Springer, 2005.

[2] I. Kaplansky, Maximal Fields with Valuation. Duke Math. J. Volume 9, Number 2 (1942), 303 - 321.

[3] J. Koenigsmann, p-Henselian Fields. Manuscripta Math. 87 (1995), no. 1, $89-99$. 
Adresses and contacts of the authors:

DMA, ENS - 45 rue d'Ulm, 75230 Paris cedex 05, FRANCE e-mail: zoe.chatzidakis@ens.fr, milan.perera@ens.fr 